Article

\title{
Avoidance and Potential Remedy Solutions of Chimeras in Reconstructing the Phylogeny of Aphids Using the 16S rRNA Gene of Buchnera: A Case in Lachninae (Hemiptera)
}

\section{Rui Chen ${ }^{1,2, \dagger}$, Zhe Wang ${ }^{1,3, \dagger}$, Jing Chen ${ }^{1}$ and Ge-Xia Qiao ${ }^{1, *}$}

1 Key Laboratory of Zoological Systematics and Evolution, Institute of Zoology, Chinese Academy of Sciences, Beijing 100101, China; E-Mails: chrui11@live.cn (R.C.); wzhe1226@126.com (Z.W.); chenjing@ioz.ac.cn (J.C.)

2 College of Life Sciences, University of Chinese Academy of Sciences, Beijing 100049, China

3 Institute of Plant Protection, Liaoning Academy of Agricultural Sciences, Shenyang 110161, China

$\dagger$ These authors contributed equally to this work.

* Author to whom correspondence should be addressed; E-Mail: qiaogx@ioz.ac.cn Tel.: +86-10-6480-7133; Fax: +86-10-6480-7099.

Academic Editor: William Chi-shing Cho

Received: 01 July 2015 / Accepted: 18 August 2015 / Published: 25 August 2015

\begin{abstract}
It is known that PCR amplification of highly homologous genes from complex DNA mixtures can generate a significant proportion of chimeric sequences. The $16 \mathrm{~S}$ rRNA gene is not only widely used in estimating the species diversity of endosymbionts in aphids but also used to explore the co-diversification of aphids and their endosymbionts. Thus, chimeric sequences may lead to the discovery of non-existent endosymbiont species and mislead Buchnera-based phylogenetic analysis that lead to false conclusions. In this study, a high probability $(6.49 \%)$ of chimeric sequence occurrence was found in the amplified 16S rRNA gene sequences of endosymbionts from aphid species in the subfamily Lachninae. These chimeras are hybrid products of multiple parent sequences from the dominant species of endosymbionts in each corresponding host. It is difficult to identify the chimeric sequences of a new or unidentified species due to the high variability of their main parent, Buchnera aphidicola, and because the chimeric sequences can confuse the phylogenetic analysis of $16 \mathrm{~S}$ rRNA gene sequences. These chimeras present a challenge to Buchnera-based phylogenetic research in aphids. Thus, our study strongly suggests that using appropriate
\end{abstract}


methods to detect chimeric 16S rRNA sequences may avoid some false conclusions in endosymbiont-based aphid research.

Keywords: chimera; 16S rRNA gene; Buchnera; phylogeny; aphids; Lachninae

\section{Introduction}

Aphidina live in association with a diverse assemblage of heritable intra-cellular bacterial endosymbionts [1-3]. The endosymbionts are categorized as either primary or secondary. Primary endosymbionts are essential for the survival of their aphid hosts [4-7]. Almost all aphids have the primary endosymbiont Buchnera aphidicola (Gammaproteobacteria: Enterobacteriales: Enterobacteriaceae), which supplies essential nutrients lacking in aphid diet [8-13]. In addition, aphids can have a series of secondary endosymbionts, such as Regiella insecticola (Enterobacteriaceae), Hamiltonella defensa (Enterobacteriaceae), Serratia symbiotica (Enterobacteriaceae) [14], Wolbachia pipientis (Alphaproteobacteria: Rickettsiales) [15], and a Sodalis-like symbiont (Enterobacteriaceae) [16]. These benefit their aphid hosts by providing protection against parasitoids, pathogens, or thermal stress, though they are generally not required for host development and reproduction [17]. These secondary symbionts inhabit a variety of tissues, such as sheath cells, hemolymph, and bacteriocytes [14].

Due to the biological importance of the endosymbionts to the aphid hosts, the relationship between endosymbionts and aphids has become a hotspot of research. Many researchers have focused on the evolutionary relationship between the aphids and endosymbionts [16,18-24]. By comparing the phylogeny of Buchnera based on the 16S rRNA gene and the phylogeny of aphids based on morphological features and the 16S rRNA gene, previous studies have indicated that Buchnera is completely concordantly evolved with its aphid hosts [12,18,25]. Based on the ages of aphid fossils, biogeographical events, and the estimated substitution rates of the 16S rRNA gene, the minimum age of Buchnera association was estimated at 160-280 million years [18]. In the phylogenetic research of aphids, Buchnera markers, especially 16S rRNA gene was widely used as the third genome due to the parallel evolution with aphid hosts, thus, is a very important marker to reconstruct the phylogenetic relationship in different taxonomic levels in aphids [13,16,18,19-22,24].

The 16S ribosomal RNA gene (hereafter 16S) has been widely used in symbiont-based research as an important marker for bacterial taxonomic and phylogenetic studies. Although sequence analysis of 16S was widely used to evaluate the diversity and identities of bacterial species in insect hosts [16,26,27], many studies have reported that $16 \mathrm{~S}$ amplicon sequencing using the PCR method can misrepresent the abundance of the microbial population because of the presence of chimeras [28-30]. Chimeric sequences are usually PCR artifacts. They are believed to occur when a prematurely terminated amplicon reanneals to foreign bacterial DNA included in the same extraction and is then copied to completion in the subsequent PCR cycles. PCR-generated chimeric sequences usually consist of two phylogenetically distinct parent sequences, as other intra-cellular bacteria are phylogenetically distinct from Buchnera. The chimeras will lead to inaccurate clustering in phylogenetic studies, and also artificially increase estimates of diversity in culture-independent surveys of microbial communities because they suggest the presence of nonexistent organisms. Prior analyses have indicated that $3.8 \%$ of the $16 \mathrm{~S}$ sequences 
from the Bacteroidetes phylum (2739 sequences) were apparently chimeric [31]. In aphids, multiple endosymbionts, such as Buchnera and other facultative symbionts, reside in one individual, creating an opportunity for producing chimeras. Furthermore, due to multiple infections and the universality of primers of $16 \mathrm{~S}$, cloning sequencing rather than direct sequencing was widely used in this kind of study [16,32]. Treating the chimeric sequences as normal sequences would result in ambiguous or even false results. However, there are so far no reports of chimeric sequences in endosymbiont-based research on Aphidina.

Here, we selected the Aphididae subfamily Lachninae for a case study. The Lachninae species are known to have a high incidence of facultative symbiont infection [16], which makes Lachninae a perfect object to study the influences of chimeric sequences in endosymbiont-based aphid research. We survey the presence of chimeras in the amplification of $16 \mathrm{~S}$ sequences of endosymbionts with universal primers. And more importantly, we evaluated the effect of these chimeras on the phylogenetic reconstruction of aphids and endosymbionts.

\section{Results}

\subsection{Prevalence and Types of Pure Sequences and Chimeras}

The Buchnera $16 \mathrm{~S}$ gene sequences from 12 Lachnine species were amplified and sequenced. Chromatograms from direct sequencing had a single peak in all nucleotide positions in two samples (Supplementary File S1), but miscellaneous peaks in 10 samples (see Supplementary File 2. These two sequences were proved to be Buchnera 16s rRNA gene sequence through BLAST tool at NCBI and manually checking. Whereas the other 10 did not bear any semblance to known sequences. In other words, only $16.7 \%(2 / 12)$ effective sequences were obtained by direct sequencing. In cloning experiments of our study, 185 positive clones were selected to be sequenced; 99 were pure Buchnera sequences, 58 were Serratia symbiotica sequences, one was Wolbachia pipientis sequence, five were Arsenophonus sp. sequences, nine were Sodalis-like symbiont sequences, one was a Regiella insecticola sequence, and 12 were suspected chimeric sequences. Examination of these suspected chimeric sequences with the grammar DECIPHER [33] and UCHIME [34] both indicated that these sequences were not chimeras. Then we manually aligned these sequences with the $16 \mathrm{~S}$ sequences of endosymbionts in aphids. We found that these suspected chimeric sequences were chimeras indeed. The occurrence frequency of chimeras was $6.49 \%$ across the Lachninae. The parental sequences of these chimeric sequences were 16S from Buchnera, Serratia symbiotica, Wolbachia pipientis, Arsenophonus sp., and a kind of Sodalis-like symbiont; mitochondrial 16S was not involved. According to the position of the parental sequence in the chimeric sequence, three types of chimeric sequences were identified, namely "BA + oe", "oe + BA", and "BA + oe + BA" ("BA" = Buchnera aphidicola "oe" = "other endosymbiont" (Figures 1 and $\mathrm{S} 1$ ). The positions of the chimera breakpoints were at different conservative regions of the $16 \mathrm{~S}$ sequences, which are most likely to be similar in phylogenetically remote bacteria. 


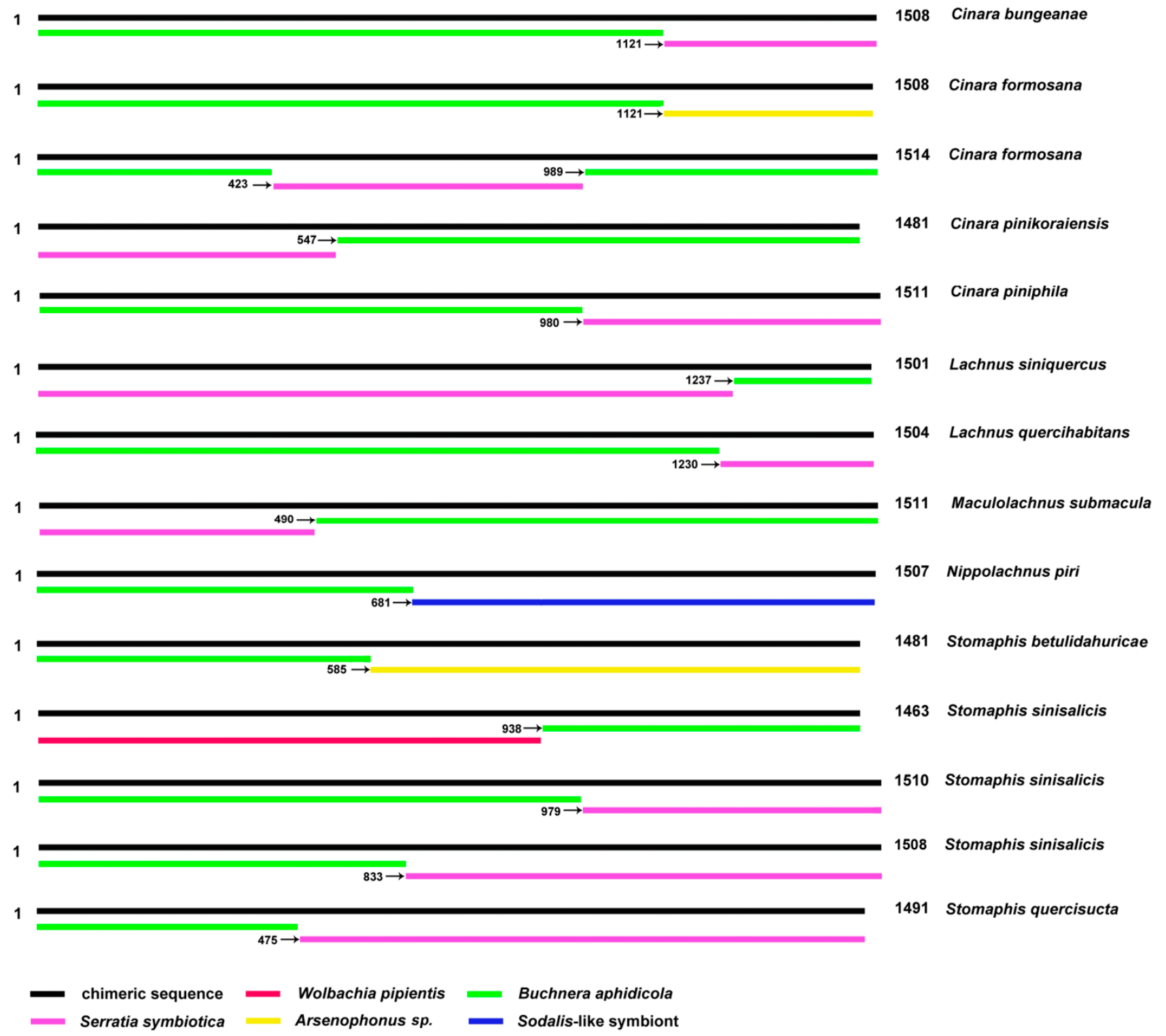

Figure 1. The types of chimeric sequences. Number at right end of bar giving length of sequence; number associated with arrow gives the sequence position where the sequence portion to the right of it starts.

\subsection{Phylogenetic Analysis of the Two Data Sets}

The results of the analyses of these two data sets indicated that the $16 \mathrm{~S}$ sequences of Buchnera clustered into five clades (red, yellow, violet, green, and gray clade in Figures 2 and 3). Tree topologies were very different for data-set II (with chimeric sequences) (Figure 3) and data-set I (Figure 2). The violet clade (= Lachninae) is monophyletic and forms the sister group of the green clade in Figure 2, while it is a "basal" paraphyletic assemblage in Figure 3. The species Tetraneura caerulescens (non-chimeric sequence) falls into the green clade in Figure 2, while in Figure 3 it is remote from this clade. All chimeric sequences fell into the in group, except for the chimeric sequence from Lachnus siniquercus (Figure 3). According to the results of the SH test, the difference between the phylogenetic trees constructed by data-sets I and II was significant because the $p$ value was $<0.05$. Thus, chimeric 
sequences can confuse the phylogenetic structure of Buchnera based on strict host correlation. We also emphasize that the ML bootstrap values are higher in data-set I than in data-set II.

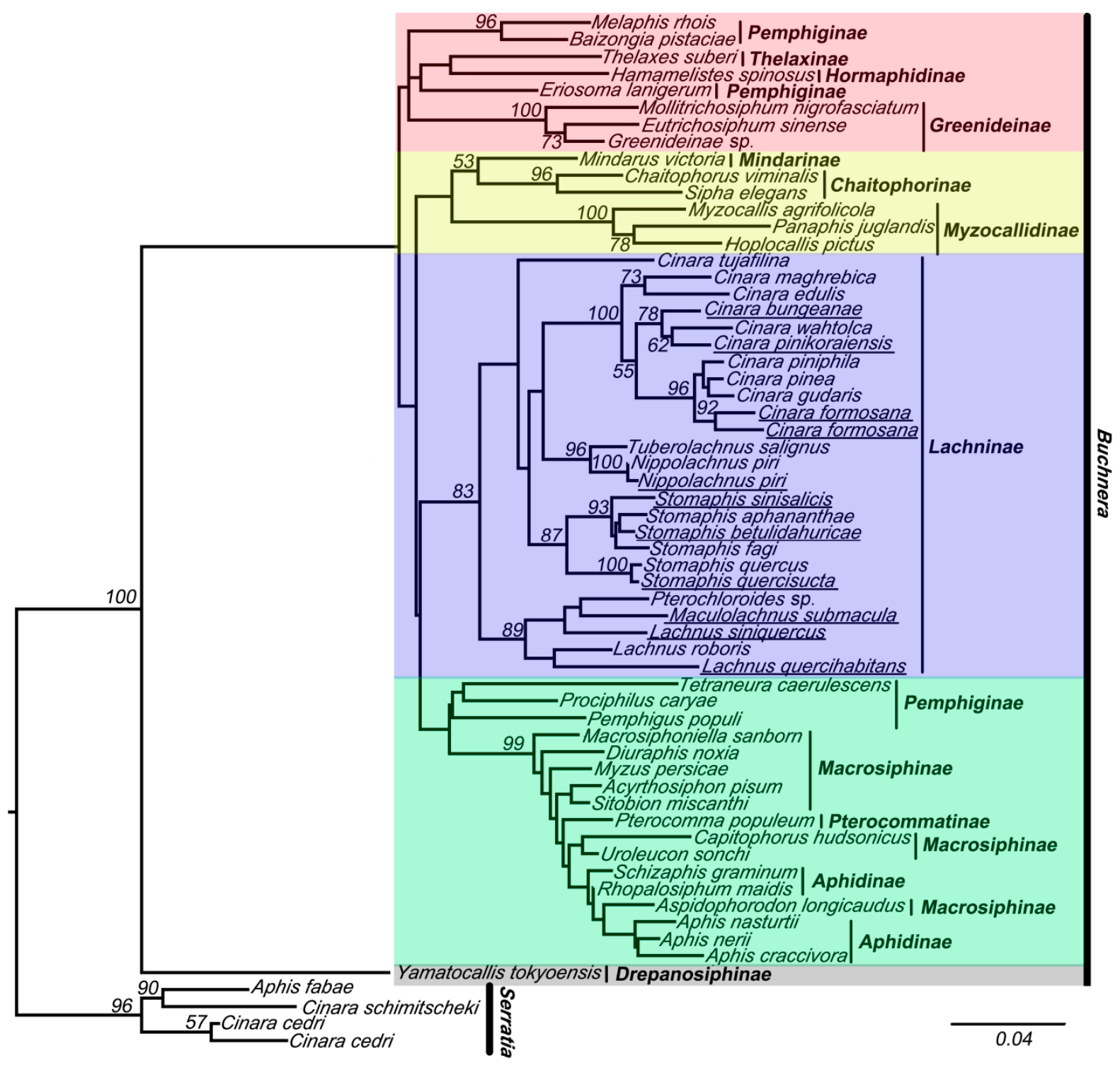

Figure 2. The maximum likelihood (ML) phylogenetic tree inferred from data-set I (without chimeric sequences). Buchnera sequences are represented by the names of their host species. The sequences obtained from this study are underlined. The nodes are marked by their ML bootstrap values. The bar represents $4 \%$ of sequence change with regard to the likelihood distance. The underlined species are with chimeric sequences. 


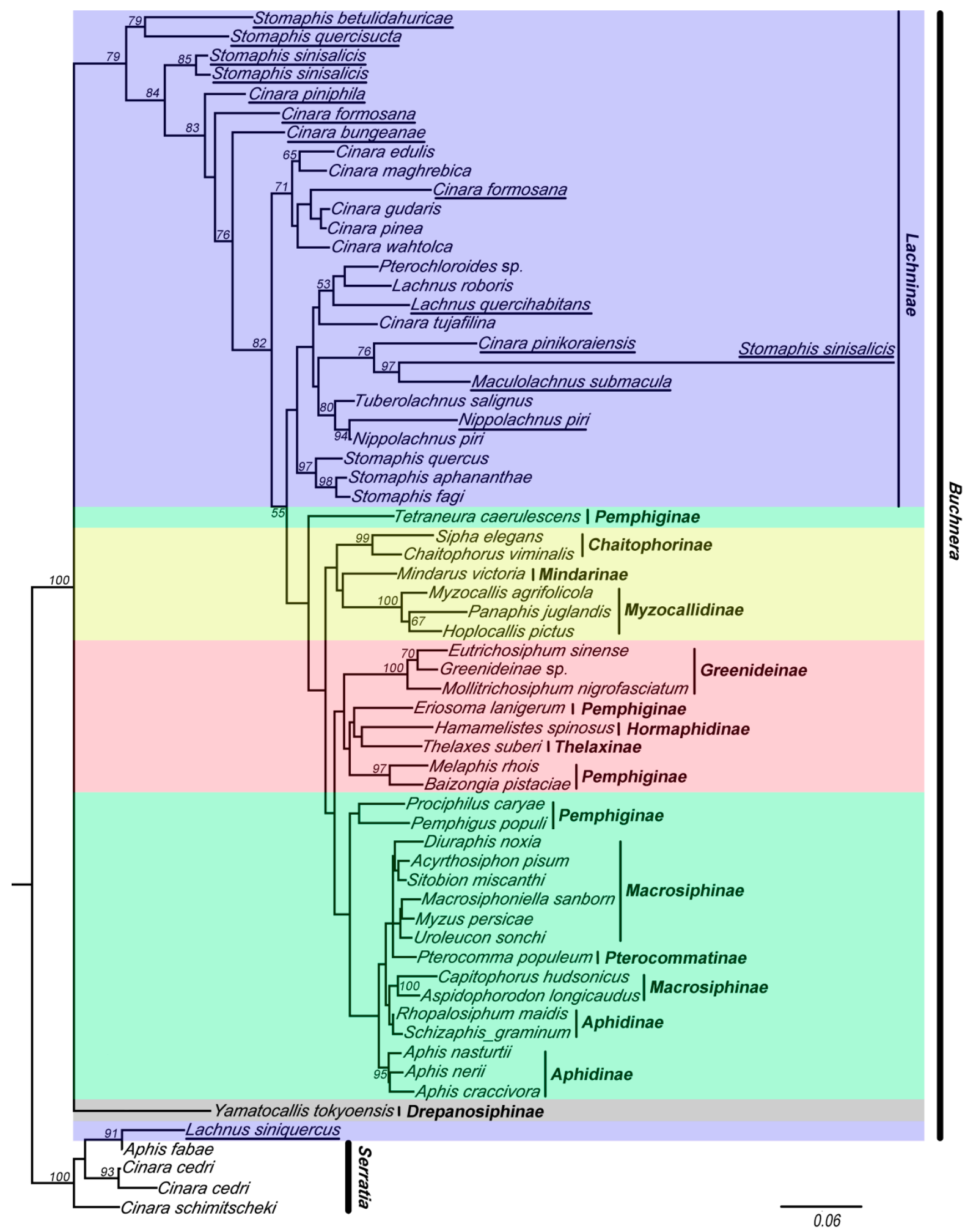

Figure 3. The maximum likelihood (ML) phylogenetic tree inferred from data-set II (with chimeric sequences, violet clade). Buchnera sequences are represented by the names of their host species. The chimeric sequences obtained from this study are underlined. The nodes are marked by their ML bootstrap values. The bar represents a $6 \%$ sequence change with regard to the likelihood distance. The underlined species are with chimeric sequences. 


\section{Discussion}

\subsection{Composition of Chimeras in Aphid Endosymbionts}

A high probability (6.49\%) of chimeric sequence occurrence was found when amplifying the 16S rRNA gene sequences of endosymbionts from Lachninae. Most chimeric sequences were formed with the aphid primary symbiont Buchnera as well as the secondary symbiont Serratia symbiotica, which was found in many Lachninae [16,35] (Figure 1). S. symbiotica may be beneficial to hosts due to their ability to supplement nutrition and to thus compensate for the inadequate provision of nutrients by Buchnera [16]. Moreover, Arsenophonus and Sodalis-like symbionts were found more likely to infect the genera Stomaphis and Nippolachnus [16]. Accordingly, the chimeric sequences from the two species Stomaphis sinisalicis and Nippolachnus piri included fragments from Buchnera, Arsenophonus, and Sodalis. In addition, Arsenophonus was found in Cinara formosana in our study, and a chimera composed of Buchnera and Arsenophonus was also found in that aphid species. Wolbachia is widely distributed in insects and was found in some species of Lachninae but not in Stomaphis [15]. In our study, Wolbachia was found in Stomaphis sinisalicis along with a chimeric sequence including Buchnera and Wolbachia fragments. All this supports that chimeras created from the predominant species of endosymbionts can in different aphid taxa form during PCR.

According to Hugenholtz and Huber [28], chimeras are commonly formed from closely related parental sequences due to sequence similarity. Here, endosymbionts of parental sequences such as Buchnera, Serratia, Arsenophonus, and Sodalis all come from Gammaproteobacteria [14], which has ten conserved regions in the $16 \mathrm{~S}$ gene sequence [36]. It is because of the existence of these conserved regions, coupled with two or more dominant symbionts living in one aphid individual, so it is likely that the obtained sequences were chimeras.

\subsection{Disguised Chimeric Sequences in Buchnera-Based Research}

The 16S rRNA gene is widely used for bacterial taxonomic and phylogenetic studies because its divergence is large enough to discriminate between varieties of bacteria [36]. For Buchnera, the divergences of the 16S rRNA sequences among different aphid host taxa are large enough for it to be used to reconstruct the phylogenetic relationships of the corresponding host taxa $[16,20,23]$. Some intraspecific divergence values of Buchnera $16 \mathrm{~S}$ sequences from different aphid species are even larger than the interspecific divergence values of different bacteria. For example, the highest divergence values of Buchnera from different aphid species in our studies reach $12.8 \%$, while bacterial lineages with more than $3 \%$ divergence of $16 \mathrm{~S}$ rRNA are recognized as distinct OTUs [37,38]. It is difficult to identify chimeric sequences from a new or unidentified aphid species as such due to the high variation of $16 \mathrm{~S}$ in Buchnera. When a new sequence of Buchnera is encountered, a search of the BLAST databases in NCBI [39] and the reconstruction of the phylogenetic tree can help to identify the species of the new sequence. However, when a BLAST search is done for a chimeric sequence, the result might indicate that the chimeric sequences are most similar to one or more sequences that may come from only one of the parent sequences identified by other research, such as Buchnera (Figure S2). Therefore, the chimeras can be misinterpreted as representing Buchnera even though they are actually from a novel aphid species. 
Likewise, when using phylogenetic reconstruction to address the sequences, chimeric sequences are usually clustered into one clade with pure Buchnera sequences (Figure 2), and chimeric sequences can be put into any position in phylogenetic tree. Moreover, in some taxa, such as Nippolachnus piri, the chimeric sequence and the normal sequence can cluster together. Thus, chimeric sequences are difficult to identify by conventional detection methods. Programs used for detecting chimeras, such as CHIMERA_CHECK in RDP [40], found the chimeras by determining whether fragments of two independent database entries had a higher overall similarity to the query sequence than a single, full-length database entry [41,42]. Unfortunately, if the parent sequences are unknown, this method is similarly unhelpful. Thus, understanding the composition of the dominant symbionts in different aphid taxa is a prerequisite to finding the parent sequences.

In our study, we used the Find Chimeras function in DECIPHER package [43] and UCHIME to find the chimeras. The software programs DECIPHER and UCHIME are widely used to checking chimera. The DECIPHER package was high-efficiency. When evaluated with the data set of simple two-parent chimeras, ss_DECIPHER and fs_DECIPHER detected $88 \%$ and $75 \%$ of the chimeras, while Uchime, ChimeraSlayer, and WigeoN detected 73\%, 56\%, and 47\%, respectively [33]. Different from DECIPHER, UCHIME is a chimera finding algorithm that uses a premise to detect the chimeras that sometimes DECIPHER did not [34]. The results of DECIPHER and UCHIME both indicated that the suspected chimeric sequences obtained in our study were not chimeras. Then we manually aligned these sequences with the $16 \mathrm{~S}$ sequences of endosymbionts in aphids. We found that these suspected chimeric sequences were chimeras. In addition, based on the phylogenetic tree, we found that the chimeras have two traits. Firstly, the chimeras in the Buchnera tree showed a tendency to be dragged far to the base of the tree (for example, chimeras from Stomaphis, Figure 3), because the chimeric sequences whose partner, for example Serratia symbiotica, was used as outgroup in our study. Secondly, chimeras should be placed further up in the tree but on very long branches (for example, chimera from Stomaphis sinisalicis).

Moreover, there were some strange aspects in the tree of data-set II (with chimeras) (Figure 3). For example, Cinara formosana (chimera without Serratia symbiotica, Figure 1) was closer to the base than Cinara bungeaneae (chimera with Serratia symbiotica); Stomaphis betulidahuricae (chimera without Serratia, Figure 1) and Stomaphis quercisucta (chimera with Serratia symbiotica) form a clade, and especially both have similar branch lengths compared to outgroup Serratia symbiotica. The reason which caused this phenomenon may be the higher difference within Buchnera. According to our analysis, the genetic distance within Buchnera was from $0.2 \%$ to $12.8 \%$; the genetic distance between Buchnera and other symbionts was: 14.2\% (Buchnera and Serratia symbiotica), 12.4\% (Buchnera and Sodalis-like symbiont), 15.0\% (Buchnera and Arsenophonus sp.), and 26.0\% (Buchnera and Wolbachia). So the branch length of chimeras which formed with Buchnera and Serratia symbiotica was similar to the outgroups, and the chimera which formed with Buchnera and Wolbachia formed a long branch clade.

\subsection{Effects of Chimeric Sequences on Buchnera-Based Research}

Our results showed that chimeric sequences affect the $16 \mathrm{~S}$ phylogeny of Buchnera. The comparisons in our study showed that the chimeric sequences confused the phylogenetic analysis of the 16S rRNA gene sequences. Although only part of these chimeric sequences came from Buchnera and the lengths of 
the Buchnera sequences within the chimeric sequences were less than half of the total sequence, these chimeras were still clustered with the Buchnera sequences, such as with the chimera from Stomaphis sinisalicis (Figure S1). The addition of these chimeras changed the relationship of the normal sequences and led to incorrect results (Figures 2 and 3). With the parallel evolution of Buchnera and its aphid hosts as demonstrated in Aphidoidea [24], many researchers focus on reconstructing the phylogeny of aphids based on the genes of Buchnera. The 16S rRNA gene was necessary in related studies. However, the chimeras will challenge Buchnera-based aphid phylogenetic research. The changes to the topology of the phylogenetic tree caused by chimeras may mislead the analysis and may lead to false conclusions.

In a recent report, the gnd gene of Buchnera combined with the mitochondrial COI gene was selected as an efficient aphid barcode [44]. It puts forward a new idea that using the gene of the endosymbiont to identify host species. In theory, the $16 \mathrm{~S}$ rRNA gene of Buchnera is also a suitable barcode marker to identify aphid due to its high-divergence among diverse host taxa. However, the high frequency of the occurrence of chimera seriously affects the utility of the 16S rRNA gene as a barcode for identifying the species. Our study strongly suggests that we should pay more attention to abnormal sequences in the alignment when the $16 \mathrm{~S}$ gene is used for endosymbiont-based aphid research; and use appropriate methods to detect chimeric 16S rRNA sequences can avoid some false conclusions in endosymbiont-based aphid research. Thus, we could reduce the effect of chimeras in the following ways: (1) improve the PCR conditions to decrease the chimeras, such as designing specific primers for different species in hypervariable regions of the $16 \mathrm{~S}$ rRNA gene; using touch down PCR to improve the specificity of PCR amplification; and using Hi Fi Taq polymerase to ensure the accuracy of the PCR amplification, although the chimeras cannot be eliminated entirely in experiments [45]; (2) defining a selection of confirmed pure $16 \mathrm{~S}$ sequences of the various bacterial taxa at GenBank (or in a separate tool), and then dividing new $16 \mathrm{~S}$ sequences within the conserved regions, and blast the portions separately against the pure sequences.

\section{Experimental Section}

\subsection{Samples, DNA Extraction, PCR, Cloning, and Sequencing}

We sampled 12 species from Aphididae-Lachninae (Table 1). Three to five individuals per sample were used as slide-mounted specimens for morphological identification. All samples were stored in 95\% or $100 \%$ ethanol and deposited in the National Zoological Museum of China, Institute of Zoology, Chinese Academy of Sciences, Beijing, China.

DNA extraction was performed with a single aphid from each sample using a DNeasy kit (Qiagen, Frankfurt, Germany). PCR was used to amplify the 16S rRNA gene sequence using the primer pair 16SF (5'-AGAGTTTGATCATGGCTCAGATTG-3') and 16SR (5'-TACCTTGTTACGACTTCACCCCAG-3'), which was designed specifically for Buchnera [46], and is widely used in studies of aphid endosymbionts $[16,20,23]$. PCR amplification was performed in a $30 \mu \mathrm{L}$ reaction volume consisting of $3.0 \mu \mathrm{L} 10 \times$ PCR buffer, $2.4 \mu \mathrm{L}$ dNTPs (10 mM each), $20 \mu \mathrm{L}$ dd $\mathrm{H}_{2} \mathrm{O}, 0.6 \mu \mathrm{L}$ of each $10 \mu \mathrm{M}$ forward and reverse primers, and one unit of TaqDNA polymerase. Every PCR included a negative control (double-distilled water instead of DNA). The PCR conditions were as follows: $95^{\circ} \mathrm{C}$ for $5 \mathrm{~min}$; 35 cycles 
consisting of denaturation at $95^{\circ} \mathrm{C}$ for $1 \mathrm{~min}, 65^{\circ} \mathrm{C}$ for $0.5 \mathrm{~min}$, and extension at $72{ }^{\circ} \mathrm{C}$ for $2 \mathrm{~min}$; and a final extension period at $72{ }^{\circ} \mathrm{C}$ for $10 \mathrm{~min}$.

Every PCR product was purified using a DNA Fragment Purification kit (TransGen, Beijing, China). Then two methods were used to obtain the sequences of every PCR products: directly sequencing and cloning. In the process of directly sequencing part of every purified PCR product was put in the sequencer and sequenced. During cloning experiments, the every other PCR purified product was ligated into the plasmid vector pMD19-T (TaKaRa, Dalian, China), and at least 20 clones from each product were sequenced on an ABI 3730 automated sequencer. Both strands of the plasmids were sequenced using universal primers (M13+, M13-) with forward and reverse reads. All sequences obtained from this study were deposited in GenBank (accession numbers in Table 1). Chimeric sequences are shown in Supplementary File S3.

\subsection{Sequence Analysis}

The sequences obtained were assembled using SeqManII of Lasergene v5.0 (DNASTAR, Madison, WI, USA) and were manually verified in DNAMAN v5.2.2.

Chimeric sequences were checked using the DECIPHER package through the use of the Find Chimeras function [43] and UCHIME in reference mode [34].

To estimate the effect of these chimeras on the phylogenetic analysis of Buchnera, two data sets were selected for analysis. Data-set I was made up of 61 normal 16S sequences of Buchnera, including 45 sequences downloaded from GenBank [16,20,24,25,47-53] (Table S1), 12 sequences from this study (Table 1), and four sequences of Serratia symbiotica (Table S2) that were defined as the outgroup [54]. The aphid hosts of Buchnera in data-set I represented 12 subfamilies of Aphididae. Most of the data in data-set II was the same as in data-set I except that in many Lachninae normal sequences obtained from our study were replaced by authentic chimeras that we identified after PCR (Supplementary Files S1 and S2). Each data set was aligned using ClustalX v1.8.3 with the default settings [55]. Shimodaira-Hasegawa (SH) tests of topology [56] were carried out using PAUP 4 b10 to assess the level of incongruence between these two data sets.

The phylogenetic analysis was conducted using the maximum likelihood (ML) method. The ML analyses were performed in RAxML7.2.8, using a heuristic search with the GTRCAT model and bootstrapped with 1000 replicates [57]. 
Table 1. The detailed collection information and GenBank accession numbers of endosymbionts of Lachninae species.

\begin{tabular}{|c|c|c|c|c|c|}
\hline Species & Location (China) & $\begin{array}{c}\text { Collection } \\
\text { Date }\end{array}$ & $\begin{array}{c}\text { No. } \\
\text { Voucher }\end{array}$ & $\begin{array}{c}\text { GB. Number } \\
\text { Buchnera }\end{array}$ & $\begin{array}{c}\text { GB. Number } \\
\text { Other Symbionts }\end{array}$ \\
\hline Cinara bungeanae (Zhang et al., 1993) [58] & Beijing & $30 . i v .2005$ & 16107 & KF751194 & KF751206 \\
\hline Cinara formosana (Takahashi, 1924) [59] & Fujian: Wuyi Mountains & 21.x.2005 & 18072 & KF751197 & KF751209 \\
\hline Cinara formosana (Takahashi, 1924) [59] & Yunnan: Lijiang City & 27.iv.2006 & 18216 & KF751198 & JN990929 \\
\hline Cinara pinikoraiensis (Zhang, 1989) [60] & Heilongjiang: Yichun City & 10.viii.2005 & 17836 & KF751196 & KF751208 \\
\hline Cinara piniphila (Ratzeburg, 1844) [61] & Inner Mongolia: HulunBuir & 13.viii.2004 & 15921 & KF751193 & KF751205 \\
\hline Lachnus quercihabitans (Takahashi, 1924) [59] & Guangxi: Lingui County & 03.xi.2010 & 26064 & KF751202 & KF751214 \\
\hline Lachnus siniquercus (Zhang, 1982) [62] & Guizhou: Leigong Mountain & 04.vi.2005 & 16278 & KF751195 & KF751207 \\
\hline Maculolachnus submacula (Walker, 1848) [63] & Xinjiang: Nilka County & 05.ix.2002 & 13796 & KF751192 & KF751204 \\
\hline Nippolachnus piri (Matsumura, 1917) [64] & Anhui: Yuexi County & 21.vii.2007 & 20199 & KF751201 & KF751213 \\
\hline Stomaphis betulidahuricae (Zhang et al., 1999) [65] & Beijing & 23.vii.2006 & 19448 & KF75200 & KF751212 \\
\hline Stomaphis quercisucta (Qiao et al., 1999) [65] & Beijing & 29.viii.2009 & Y8896 & KF751203 & KF751215 \\
\hline Stomaphis sinisalicis (Zhang et al., 1982) [62] & Beijing & 15.vi.2006 & 19106 & KF751199 & $\begin{array}{l}\text { KF751210/ } \\
\text { KF751211 }\end{array}$ \\
\hline
\end{tabular}




\section{Conclusions}

Our research indicated that chimeric sequences were usually obtained when amplifying 16S rRNA gene sequences of endosymbionts from aphid species. It is difficult to identify the chimeric sequences from a new or unidentified species. These chimeric sequences always confuse the phylogenetic analysis of 16S rRNA gene sequences. So we must pay attention to the chimera when studying endosymbionts in aphids, and identify the chimera according to the ways proposed in this article.

\section{Supplementary Materials}

Supplementary materials can be found at http://www.mdpi.com/1422-0067/16/09/20152/s1.

\section{Acknowledgments}

We want to thank $\mathrm{Li} \mathrm{Hu}$ and Cui Ying for the help of data analysis and viewpoints discussion with this review. The work was supported by National Natural Sciences Foundation of China (Nos. 31430078, 31372237), the External Cooperation Program of Chinese Academy of Sciences (No. 152111KYSB20130012), the Ministry of Science and Technology of the People's Republic of China (MOST Grant Nos. 2011FY120200, 2014FY210200), and National Science Fund for Fostering Talents in Basic Research (No. J1210002).

\section{Author Contributions}

Author Rui Chen and author Zhe Wang performed most of the laboratory and analytical work, and participated in conceptual design and manuscript preparation; author Jing Chen participated in conceptual design; author Ge-Xia Qiao coordinated the study, performed most of the identifications, and participated in conceptual design. All authors contributed to revising the manuscript.

\section{Conflicts of Interest}

The authors declare no conflict of interest.

\section{References}

1. Buchner, P. Endosymbiosis of Animals with Plant Microorganisms; Interscience Publishers: New York, NY, USA, 1965.

2. Dasch, G.A.; Weiss, E.; Chang, K.P. Endosymbionts of insects. In Bergey's Manual of Systematic Bacteriology; Krieg, N.R., Holt, J.G., Eds.; Williams and Wilkins: Baltimore, MD, USA, 1984; pp. 811-833.

3. Campbell, B.C. On the role of microbial symbiotes in herbivorous insects. In Insect-Plant Interactions; Bernays, E.A., Ed.; CRC Press: Boca Raton, FL, USA, 1990; pp. 1-44.

4. Blackman, R.L. Reproduction, cytogenetics, and development. In Aphids: Their Biology, Natural Enemies, and Control; Minks, A.K., Harrewijn, P., Eds.; Elsevier Biomedical Press: Amsterdam, The Netherlands, 1987; pp. 163-195.

5. Douglas, A.E. Mycetocyte symbiosis in insects. Biol. Rev. 1989, 64, 409-434. 
6. Houk, E.J.; Griffiths G.W. Intracellular symbiotes of the Homoptera. Annu. Rev. Entomol. 1980, 25, 161-187.

7. Ishikawa, H. Biochemical and molecular aspects of endosymbiosis in insects. Int. Rev. Cytol. 1989, $116,1-45$.

8. Untermann, B.M.; Baumann, P.; Mclean, D.L. Pea aphid symbiont relationships established by analysis of 16S rRNAs. J. Bacteriol. 1989, 171, 2970-2974.

9. Munson, M.A.; Baumann, P.; Kinsey, M.G. Buchnera gen. nov. and Buchnera aphidicola sp. nov., a taxon consisting of the mycetocyte associated, primary endosymbionts of aphids. Int. J. Syst. Bacteriol. 1991, 41, 566-568.

10. Douglas, A.E.; Prosser, W.A. Synthesis of the essential amino acid tryptophan in the pea aphid (Acyrthosiphon pisum) symbiosis. J. Insect Physiol. 1992, 38, 565-568.

11. Baumann, P.; Baumann, L.; Lai, C.Y.; Rouhbaksh, D.; Moran, N.A.; Clark, M.A. Genetics, physiology and evolutionary relationships of the genus Buchnera: Intracellular symbionts of aphids. Ann. Rev. Microbiol. 1995, 49, 55-94.

12. Baumann, P.; Moran, N.A.; Baumann, L. The evolution and genetics of aphid endosymbionts. BioScience 1997, 47, 12-20.

13. Nakabachi, A.; Ishikawa, H. Differential display of mRNAs related to amino acid metabolism in the endosymbiotic system of aphids. Insect Biochem. Mol. Biol. 1997, 27, 1057-1062.

14. Oliver, K.M.; Degnan, P.H.; Burke, G.R.; Moran, N.A. Facultative symbionts in aphids and the horizontal transfer of ecologically important traits. Annu. Rev. Entomol. 2010, 55, 247-266.

15. Augustinos, A.A.; Santos-Garcia, D.; Dinoyssopoulou, E.; Moreira, M.; Papapanagotou, A.; Scarvelakis, M.; Doudoumis, V.; Khadem, M.; Latorre, A.; Tsiamis, G.; et al. Detection and characterization of Wolbachia infections in natural populations of aphids: Is the hidden diversity fully unraveled? PLOS ONE 2011, 6, e28695.

16. Burke, G.R.; Normark, B.B.; Favret, C.; Moran, N.A. Evolution and diversity of facultative symbionts from the aphid subfamily Lachninae. Appl. Environ. Microbiol. 2009, 75, 5328-5335.

17. Feldhaar, H. Bacterial symbionts as mediators of ecologically important traits of insect hosts. Ecol. Entomol. 2011, 36, 533-543.

18. Moran, N.A.; Munson, M.A.; Baumann, P.; Ishikawa, H. A molecular clock in endosymbiotic bacteria is calibrated using the insect hosts. Proc. R. Soc. Lond. B 1993, 253, 167-171.

19. Clark, M.A.; Moran, N.A.; Baumann, P.; Wernegreen, J.J. Cospeciation between bacterial endosymbionts (Buchnera) and are recent radiation of aphids (Uroleucon) and pitfalls of testing for phylogenetic congruence. Evolution 2000, 54, 517-525.

20. Martinez-Torres, D.; Buades, C.; Latorre, A.; Moya, A. Molecular systematics of aphids and their primary endosymbionts. Mol. Phylogenet. Evol. 2001, 20, 437-449.

21. Pérez-Brocal, V.; Gil, R.; Ramos, S.; Lamelas, A.; Postigo, M.; Michelena, J.M.; Silva, F.J.; Moya, A.; Latorre, A. A small microbial genome: The end of a long symbiotic relationship? Science 2006, 314 , 312-313.

22. Jousselin, E.; Desdevises, Y.; Coeur d'acier, A. Fine-scale cospeciation between Brachycaudus and Buchnera aphidicola: Bacterial genome helps define species and evolutionary relationships in aphids. Proc. R. Soc. Lond. B 2009, 276, 187-196. 
23. Liu, L.; Huang, X.L.; Zhang, R.L.; Jiang, L.Y.; Qiao, G.X. Phylogenetic congruence between Mollitrichosiphum (Aphididae: Greenideinae) and Buchnera indicates insect-bacteria parallel evolution. Syst. Entomol. 2013, 38, 81-92.

24. Nováková, E.; Hypša, V.; Klein, J.; Foottit, R.G.; von Dohlen, C.D.; Moran, N.A. Reconstructing the phylogeny of aphids (Hemiptera: Aphididae) using DNA of 3 the obligate symbiont Buchnera aphidicola. Mol. Phylogenet. Evol. 2013, 68, 12-54.

25. Munson, M.A.; Baumann, P.; Clark, M.A.; Baumann, L.; Moran, N.A.; Voegtlin, D.J.; Campbell, B.C. Evidence for the establishment of aphid-eubacterium endosymbiosis in an ancestor of four aphid families. J. Bact. 1991,173, 6321-6324.

26. Clarridge, J.E.; Jill, E. Impact of $16 \mathrm{~S}$ rRNA gene sequence analysis for identification of bacteria on clinical microbiology and infectious diseases. Clin. Microbiol. Rev. 2004, 17, 840-862.

27. Doudoumis, V.; Tsiamis, G.; Wamwiri, F.; Brelsfoard, C.; Alam, U.; Aksoy, E.; Dalaperas, S.; Abd-Alla, A.; Ouma, J.; Takac, P.; et al. Detection and characterization of Wolbachia infections in laboratory and natural populations of different species of tsetse flies (genus Glossina). BMC Microbiol. 2012, 12, doi:10.1186/1471-2180-12-S1-S3.

28. Hugenholtz, P.; Huber, T. Chimeric 16S rDNA sequences of diverse origin are accumulating in the public databases. Int. J. Syst. Evol. Microbiol. 2003, 53, 289-293.

29. Gonzalez, J.M.; Zimmermann, J.; Saiz-Jimenez, C. Evaluating putative chimeric sequences from PCR-amplified products. Bioinformatics 2005, 21, 333-337.

30. Schloss, P.D.; Gevers, D.; Westcott, S.L. Reducing the effects of PCR amplification and sequencing artifacts on 16S rRNA-based studies. PLoS ONE 2011, 6, e27310.

31. Ashelford, K.E.; Chuzhanova, N.A.; Fry, J.C.; Jones, A.J.; Weightman, A.J. At least 1 in 20 $16 \mathrm{~S}$ rRNA sequence records currently held in public repositories is estimated to contain substantial anomalies. Appl. Environ. Microbiol. 2005, 71, 7724-7736.

32. Von Dohlen, C.D.; Spaulding, U.; Shields, S.; Havill, N.P.; Rosa, C.; Hoover, K. Diversity of proteobacterial endosymbionts in hemlock woolly adelgid (Adelges tsugae) (Hemiptera, Adelgidae) from its native and introduced range. Environ. Microbiol. 2013, 15, 2043-2062.

33. Wright, E.S.; Yilmaz, L.S.; Noguera, D.R. DECIPHER, A Search-Based approach to chimera identification for 16S rRNA sequences. Appl. Environ. Microbiol. 2012, 78, 717.

34. Edgar, R.C.; Haas, B.J.; Clemente, J.C.; Quince, C.; Knight, R. UCHIME improves sensitivity and speed of chimera detection. Bioinformatics 2011, 27, 2194-2200.

35. Lamelas, A.; Pérez-Brocal, V.; Gómez-Valero, L.; Gosalbes, M.; Moya, A.; Latorre, A. Evolution of the secondary symbiont "Candidatus Serratia symbiotica" in aphid species of the subfamily Lachninae. Appl. Environmen. Microbiol. 2008, 74, 4236-4240.

36. Chakravorty, S.; Helb, D.; Burday, M.; Connell, N.; Alland, D. A detailed analysis of 16S ribosomal RNA gene segments for the diagnosis of pathogenic bacteria. J. Microbiol. Methods 2007, 69, 330-339.

37. Stackebrandt, E.; Goebel, B.M. Taxonomic note: A place for DNA-DNA reassociation and 16S rRNA sequence analysis in the present species definition in bacteriology. Int. J. Syst. Bacteriol. 1994, 44, 846-849.

38. Ratnasingham, S.; Hebert, P.D.N. A DNA-based registry for all animal species: The Barcode Index Number. PLoS ONE 2013, 8, e66213. 
39. National Center of Biotechnology Information (NCBI). Available online: http://blast.ncbi.nlm. nih.gov/Blast.cgi (accessed on 1 August 2014).

40. Maidak, B.L.; Cole, J.R.; Lilburn, T.G.; Parker, C.T.; Saxman, P.R.; Farris, R.J; Garrity, G.M.; Olsen, G.J.; Schmidt, T.M.; Tiedje, J.M. The RDP-II (Ribosomal Database Project). Nucleic Acids Res. 2001, 29, 173-174.

41. Komatsoulis, G.; Waterman, M. A new computational method for detection of chimeric 16S rRNA artifacts generated by PCR amplification from mixed bacterial populations. Appl. Environ. Microbiol. 1997, 63, 2338-2346.

42. Robinson-Cox, J.F.; Bateson, M.M.; Ward, D.M. Evaluation of nearest-neighbor methods for detection of chimeric small subunit rRNA sequences. Appl. Environ. Microbiol. 1995, 61, 1240-1245.

43. Database Enabled Code for Ideal Probe Hybridization Employing R (DECIPHER). Available online: http://decipher.cee.wisc.edu/FindChimeras.html) (accessed on 16 October 2014).

44. Chen. R.; Jiang, L.Y.; Liu, L.; Liu, Q.H.; Wen, J.; Zhang, R.L.; Li, X.Y.; Wang, Y.; Lei, F.M.; Qiao, G.X. The gnd gene of Buchnera as a new, effective DNA barcode for aphid identification. Syst. Entomol. 2013, 38, 615-625.

45. Haas, B.J.; Gevers, D.; Earl, A.M.; Feldgarden, M.; Ward, D.V.; Giannoukos, G.; Ciulla, D.; Tabbaa, D.; Highlander, S.K.; Sodergren, E.; et al. Chimeric 16S rRNA sequence formation and detection in Sanger and 454-pyrosequenced PCR amplicons. Genome Res. 2011, 21, 494-504.

46. Van Ham, R.C.H.J.; Moya, A.; Latorre, A. Putative evolutionary origin of plasmids carrying the genes involved in leucine biosynthesis in Buchnera aphidicola (endosymbiont of aphids). J. Bacteriol. 1997, 179, 4768-4777.

47. Fukatsu, T. Secondary intracellular symbiotic bacteria in aphids of the genus Yamatocallis (Homoptera: Aphididae: Drepanosiphinae). Appl. Environ. Microbiol. 2001, 67, 5315-5320.

48. Li, Z.X.; Li, D.X. Molecular identification of the primary and secondary symbionts of green peach aphid, Myzus persicae (Sulzer). Acta Entomol. Sin. 2005, 48, 810-814.

49. Li, T.; Xiao, J.H.; Wu, Y.Q.; Huang, D.W. Diversity of bacterial symbionts in populations of Sitobion miscanthi (Hemiptera: Aphididae) in China. Environ. Entomol. 2014, 43, 605-611.

50. Shigenobu, S.; Watanabe, H.; Hattori, M.; Sakaki, Y.; Ishikawa, H. Genome sequence of the endocellular bacterial symbiont of aphids Buchnera sp. APS. Nature 2000, 407, 81-86.

51. Tamas, I.; Klasson, L.; Canback, B.; Naslund, A.K.; Eriksson, A.S.; Wernegreen, J.J.; Sandstrom, J.P.; Moran, N.A.; Andersson, S.G. 50 million years of genomic stasis in endosymbiotic bacteria. Science 2002, 296, 2376-2379.

52. van Ham, R.C.; Kamerbeek, J.; Palacios, C.; Rausell, C.; Abascal, F.; Bastolla, U.; Fernandez, J.M.; Jimenez, L.; Postigo, M.; Silva, F.J.; et al. Reductive genome evolution in Buchnera aphidicola. Proc. Natl. Acad. Sci. USA. 2003, 100, 581-586.

53. Sabri, A.; Leroy, P.; Haubruge, E.; Hance, T.; Frere, I.; Destain, J.; Thonart, P. Isolation, pure culture and characterization of Serratia symbiotica sp. nov., the R-type of secondary endosymbiont of the black bean aphid Aphis fabae. Int. J. Syst. Evol. Microbiol. 2011, 61, 2081-2088.

54. Husník, F.; Chrudimský, T.; Hypša, V. Multiple origins of endosymbiosis within the Enterobacteriaceae (g-Proteobacteria): Convergence of complex phylogenetic approaches. BMC Biol. 2011, 9, doi:10.1186/1741-7007-9-87. 
55. Thompson, J.D.; Gibson, T.J.; Plewniak, F.; Jeanmougin, F.; Higgins, D.G. The CLUSTAL_X windows interface: Flexible strategies for multiple sequence alignment aided by quality analysis tools. Nucleic Acids Res. 1997, 25, 4876-4882.

56. Shimodaira, H.; Hasegawa, M. Multiple comparisons of log-likelihoods with applications to phylogenetic inference. Mol. Biol. Evol. 1999, 16, 1114.

57. Stamatakis, A.; Hoover, P.; Rougemont, J. A rapid bootstrap algorithm for the RAxML web servers. Syst. Biol. 2008, 57, 758-771.

58. Zhang, G.X.; Zhang, W.Y.; Zhong, T.S. Studies on Chinese species of Cinara Curtis and descriptions of new species (Homoptera: Lachnidae). Sinozoologia 1993, 10, 121-141.

59. Takahashi, R. Aphididae of Formosa. Rep. Dep. Agric. Gov. Res. Inst. Formosa 1924, 10, 1-121.

60. Zhang, G.X.; Zhong, T.S. Three new species of Cinara from Northeast China (Homoptera: Aphidoidea). Acta Zootaxonom. Sin. 1989, 14, 198-204

61. Ratzeburg, J.T.C.; Schnabelkerfe, H.H.; Linn, H. Rhynchota F. In systematischer Folge und mit besonderer Rücksicht auf die Vertilgung der Schädlichen. In Die Forst-Insecten oder Abbildung und Beschreibung der in den Wäldern Preussens und der Nachbar-staaten als schädlich oder nützlich bekannt gewordenen Insecten; Fünfte, O., Ed.; Nicolaischen Buchhandlung: Berlin, Germany, 1844; pp. 1-314.

62. Zhang, G.X.; Zhong, T.S. New species and subspecies of Chinese Aphidoidea. Sinozoologia 1982, 2, 19-28.

63. Walker, F. Descriptions of Aphides. Ann. Mag. Natur. Hist. 1848, 2, 95-109.

64. Matsumura, S. A list of the Aphididae of Japan, with description of new species and genera. J. Coll. Agr. Tokoku Imp. Univ. 1917, 7, 351-414.

65. Qiao, G.X.; Zhang, G.X. A revision of Stomaphis Walker from China with descriptions of three new species (Homoptera: Lachnidae). Ent. Sin. 1999, 6, 289-298.

(C) 2015 by the authors; licensee MDPI, Basel, Switzerland. This article is an open access article distributed under the terms and conditions of the Creative Commons Attribution license (http://creativecommons.org/licenses/by/4.0/). 